\title{
Dust Structure around two Asymptotic Giant Stars at Latitude $32^{\circ} \& 40.67^{\circ}$
}

\author{
A.K. Jha and D.R.Upadhyay \\ Central Department of Physics, Tribhuvan University. Kathmandu, Nepal \\ Email:astroajay123@gmail.com
}

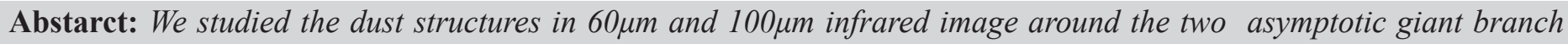
(AGB) stars. A systematic search of dust structure in the far infrared (100 $\mu \mathrm{m}$ and $60 \mu \mathrm{m})$ Infrared Astronomical Satellite (IRAS) survey was performed using Sky View Observatory. In order to find the possible candidate of cavity structure not yet studied, we used SIMBAD database to locate discrete sources in the region. A new relatively symmetric spherical cavity like structure (size: $1.354 p c \times 0.971$ pc) for candidate I and (size: $1.424 p c \times 0.925 p c$ ) for candidate II at R.A.(J2000) $=04^{h} 46^{m} 13.84^{s}$, Dec. $(J 2000)=+32^{\circ} 31^{\prime} 39.6^{\prime \prime}$ and $05^{h} 05^{m} 59.58^{s} ;+40^{\circ} 40^{\prime} 33.4^{\prime \prime}$ respectively was found at the distance of about $280 \mathrm{pc}$ and $390 \mathrm{pc}$. In this present work we have calculated the dust color temperature, mass, outflow velocity, energy and size. We also studied the flux density variation and then calculated temperature and mass profile of the dust and outflow nature of $A G B$ wind using data reduction software's Aladin v2.5 and Aladinv8.0. Our aim was to test how outflow can be seen in this region. We have studied two cavity-like structures. The dust color temperature is found to lie in the range $21.7 \mathrm{~K}$ to $35.6 \mathrm{~K}$ for candidate $\mathrm{I}$ and $18.5 \mathrm{~K}$ to $19.3 \mathrm{~K}$, with an offset of about $14 \mathrm{~K}$ and $0.8 \mathrm{~K}$ respectively. An offset of $14 \mathrm{~K}$ suggests that the $A G B$ is hot in early $A G B$ phase now in late AGB stage. We expect deviation from symmetrical outflow, i.e., $A G B$ wind probably prefer polytropic behaviour implies bipolar type wind whereas the offset 0.8 $K$ for candidate II suggests that the AGB is in the symmetric AGB phase, i.e., early AGB stage. The total mass of the dust in the cavity structure is found about $5.93 \times 10^{25} \mathrm{Kg}\left(0.00003 M_{\Theta}\right)$ for candidate I and $1.95 \times 10^{27} \mathrm{Kg}\left(0.001 M_{\Theta}\right)$ for candidate II. In addition we have calculated outflow energy of C-rich AGB star along ISM using outflow velocity of our candidates. It is found that the outflow velocities are $12.96 \mathrm{~ms}^{-1}$ and $10.71 \mathrm{~ms}^{-1}$ respectively. For this we have calculated the value of speed of sound $\left(C_{s}\right)$ and escape velocity $\left(u_{e s}\right)$. In this way we have determined outflow energy, i.e., $1.173 \times 10^{25} \mathrm{~J}$ and 2.766 $\times 10^{27} \mathrm{~J}$ respectively.
\end{abstract}

Key words: Interstellar medium, Asymptotic giant branch, AGB wind, Polytropes.

\section{INTRODUCTION}

Low intermediate mass star $(1-8 \mathrm{M} \odot)$ are very important contributors of material to the interstellar medium (ISM), and yet the mechanisms by which this matter is expelled remain a mystery. In this research work we discuss how mass distributed near AGB star, temperature profile, shock nature of AGB star near cavity like structure plays a role in studying the interaction between ISM and AGB star at given latitude. LIMS evolve they become Asymptotic Giant Branch stars: luminous $\left(\mathrm{L}_{\star} 10^{4} \mathrm{~L}_{\odot}\right)$, cool $\left(\mathrm{T}_{\text {eff }} 3000 \mathrm{~K}\right)$ giants $\left(\mathrm{R}_{\star} 1 \mathrm{AU}\right)$, which lose mass at high rates $\left(10^{-7}\right.$ to a few times $10^{-4} \mathrm{M}_{\star} / \mathrm{yr}$ ) ( Iben et al. 1983). AGB stars pulsate due to dynamical instabilities, leading to intensive mass loss and the formation of a circumstellar shell of gas. Pulsations levitate atmospheric material allowing it to achieve escape velocity. As the gaseous material drifts away from the star it cools and molecules can form, followed by the formation of small particles (dust grains), leading to a circum stellar envelope of dust and gas. The dust grains tap into the tremendous luminosity power of the star and drive a radiation-pressured wind (Hoefner et al. 1997). This wind causes AGB stars to lose mass at such tremendous rates that they wither into white dwarfs rather than explode as supernovae Generally, the mass-loss rate, $\dot{M}$, increases over time as an AGB star evolves, and ends in an episode of extremely high mass loss, the super wind (SW) phase (Bloecker et al. 1991).

During the SW phase $\mathrm{M}$ exceeds $10^{-5} \mathrm{M}_{\star} \mathrm{yr}^{-1}$. Asymptotic giant branch (AGB) stars are generally classified as oxygen-rich (M-type) or carbon-rich (C-type) based on the chemistry of the photosphere and/or the outer envelope (Iben et al. 1983).

\subsection{Asymptotic Giant Branch stars}

As Low and intermediate mass stars $\left(1-8 \mathrm{M}_{\odot}\right)$ evolve they become Asymptotic Giant Branch (AGB) (Iben et al. 1983) stars: luminous $\left(\mathrm{L}_{\star} 10^{4} \mathrm{~L}_{\odot}\right)$, cool $\left(\mathrm{T}_{\text {eff }} 3000 \mathrm{~K}\right)$ giants $\left(\mathrm{R}_{\star} 1 \mathrm{AU}\right)$, which lose mass at high rates $\left(10^{7}\right.$ to a few times $10^{4} \dot{\mathrm{M}} / \mathrm{yr}$ ). Matter lost by AGB stars (through both the wind 
and the super wind) is enriched by various nuclear-burning products, and therefore AGB stars can play an important role in galactic nucleosynthesis. More specifically, the envelope composition of AGB stars has been influenced by up to three dredge up processes and by the EB process. Further, CN-cycled materials could have been brought to the surface by additional slow mixing processes operating on the main sequence. AGB stars pulsate due to dynamical instabilities, leading to intensive mass loss and the formation of a circumstellar shell of gas. Pulsations levitate atmospheric material allowing it to achieve escape velocity. As the gaseous material drifts away from the star it cools and molecules can form, followed by the formation of small particles (dust grains), leading to a circumstellar envelope of dust and gas. The dust grains tap into the tremendous luminosity power of the star and drive a radiation-pressured wind ( Hoefner et al. 1997). This wind causes AGB stars to lose mass at such tremendous rates that they wither into white dwarfs rather than explode as supernovae Generally, the mass-loss rate, $\dot{M}$, increases over time as an AGB star evolves, and ends in an episode of extremely high mass loss, the super wind (SW) phase ( Bloecker et al., 1991) During the SW phase $\dot{\mathrm{M}}$ exceeds $10-5 \mathrm{M}_{\odot} \mathrm{yr}^{-1}$. Continued AGB star mass loss causes the dust shell to increase in depth both optically and geometrically as mass-loss rate increases. As these stars approach the SW phase they become invisible at optical wavelengths and very IR bright. During this SW stage, intense mass loss depletes the remaining hydrogen in the star's outer envelope, and terminates the AGB phase. The rapid depletion of material from the outer envelope of the star means that this extremely high mass-loss phase must have a relatively short duration (Bloecker et al. 1991) During their ascent of the AGB, these stars also evolve chemically, starting with oxygen-rich atmospheres. Helium burning forms ${ }^{12} \mathrm{C}$, which is dredged up to the stellar surface by strong convection currents in the mantle. Thus, carbon is injected into the stellar atmosphere. The stability of the $\mathrm{CO}$ molecule in the stellar atmosphere means that the carbon-tooxygen ratio $(\mathrm{C} / \mathrm{O})$ controls the chemistry around the star: whichever element is less abundant will be entirely locked into $\mathrm{CO}$ molecules, leaving the more abundant element to control dust formation. Therefore AGB stars can be either oxygen - rich or carbon - rich (Padmanabhan et al. 2010).

\subsection{C-rich stars}

The main components of dust in the envelopes around carbon stars are believed to be featureless amorphous carbon (AMC) grains and $\mathrm{SiC}$ grains, which produce the $11.3-\mu \mathrm{m}$ emission feature. The carbon stars showing the 11.3- $\mu \mathrm{m}$ emission feature belong to IRASLRS class C. For C-rich AGB stars, Suh et al. use the same catalogue of (Suh et al. 2009) except that one reference is added and six objects are excluded in this work (Suh et al. 2009).

\section{REGION OF INTEREST}

We adopted the following steps in order to find an isolated dust structure around a low mass AGB Star. Dust structure at 100 micron around the C-rich AGB stars has systematically searched using the catalog K. W.

Suh \& Y. J. Kwon (2011). For this, we used Sky view Virtual Observatory. The isolated cavity-like structure having size $\sim 0.5^{\circ}$ is taken as a preference. In addition, the selected preferred cavity is taken nearly rounded or symmetrical in shape. The discrete sources around the cavity has studied using SIMBAD (http://simbad.u-strasbg.fr/simbad/). These structures has been looked critically. Finally best two structures in which the flux density has minimum value in the cavity has selected for further study. Fig. 1 shows 100 micron image of two selected dust structures. The location of AGB stars in Fig. 1a and b are RA(J2000) $=04^{\mathrm{h}} 46^{\mathrm{m}} 09^{\mathrm{s}}$, Dec $(\mathrm{J} 2000)=+32^{\circ} 28^{\prime} 00^{\prime \prime}$ (Candidate I, hereafter $)$; and $\operatorname{RA}(\mathrm{J} 2000)=05^{\mathrm{h}} 06^{\mathrm{m}} 02^{\mathrm{s}}, \operatorname{Dec}(\mathrm{J} 2000)$ $=+40^{\circ} 40^{\prime} 00^{\prime}$, (Candidate II, hereafter) respectively. In both structures, minimum flux density is found at $\mathrm{RA}(\mathrm{J} 2000)=$ $04^{\mathrm{h}} 46^{\mathrm{m}} 13.84^{\mathrm{s}}$, Dec (J2000) $=+32^{\circ} 31^{\prime}$ '39.6"; and RA(J2000) $=05^{\mathrm{h}} 05^{\mathrm{m}} 59.58^{\mathrm{s}}, \operatorname{Dec}(\mathrm{J} 2000)=+40^{\circ} 40^{\prime} 33.4^{\prime \prime}$, respectively. The distance to these structures are $280 \mathrm{pc}$ and $390 \mathrm{pc}$. This distance has been estimated by using star-count method and color-color/color-magnitude diagram (Odenwald,1988). 

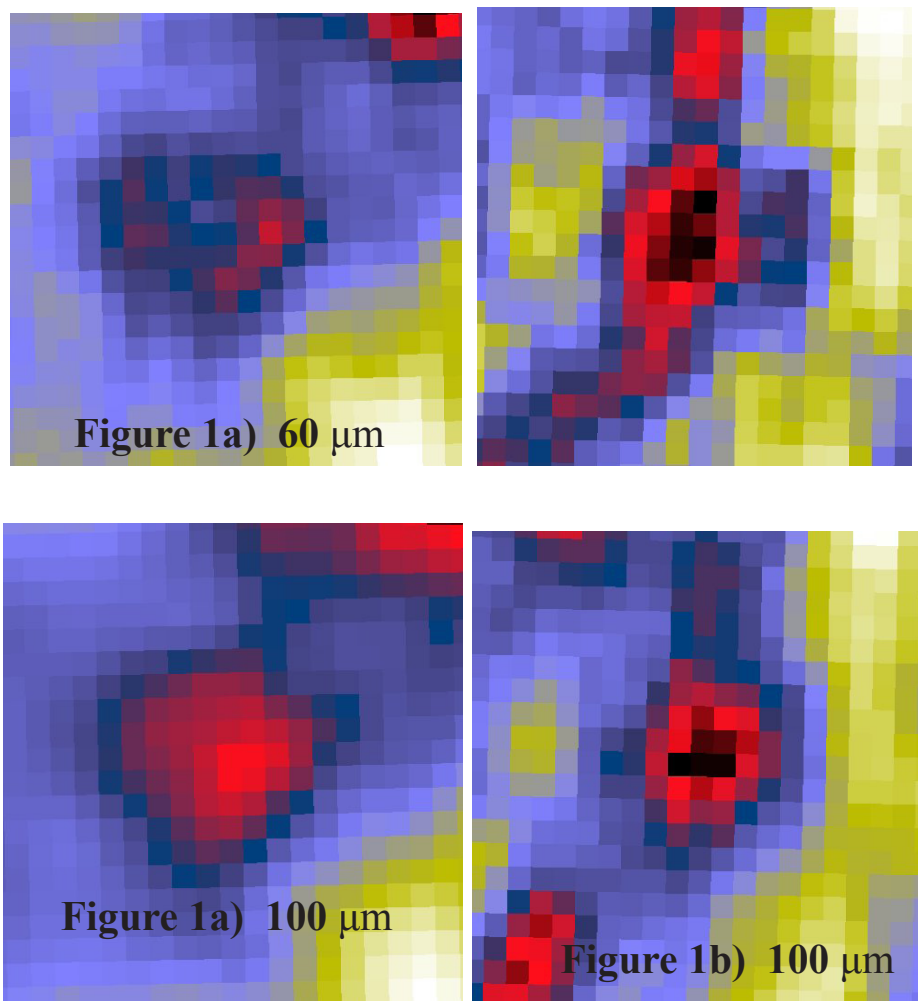

Figure 1: $0.5^{\circ} 0.5^{\circ}$ IRAS image of our region of interest in 60 $\mu \mathrm{m}$, and $100 \mu \mathrm{m}$ bands. In all bands, fine cavity including $A G B$ stars Left 1a) for R.A./Dec. (J2000): 04h 46 $09^{\mathrm{s}} /+32^{\circ} 28^{\prime} 00^{\prime \prime}$ and Right 1b) for R.A./Dec.(J2000): $05^{h} 06^{m} 02^{s} /+40^{\circ} 40^{\prime} 00^{\prime \prime}$ (SkyView, 2014).

\section{METHODS}

We adopt the following methods to find the size, dust color temperature and dust mass of the structure. In addition, we calculated speed of the AGB wind.

\subsection{Dust Color temperature Estimation}

We adopt the method developed by Schnee et al. (2005) to calculate the dust color temperature from the IRAS $60 \mu \mathrm{m}$ and $100 \mu \mathrm{m}$ flux densities (Schnee et al. 2005). For dust color temperature estimation we use the expression as

$T_{d}=-96 \frac{1}{\ln \left\{R \times 0.6^{(3+\beta)}\right\}}$

The value of the spectral emissivity index depends on dust grain properties as composition, size, and compactness. For reference, a pure blackbody would have $\beta=0$, the amorphous layer-lattice matter has $\beta \sim 1$, and the metals and crystalline dielectrics have $\beta \sim 2$. ( Dupac et al. 2003)

where $\mathrm{R}$ is given by

$\mathrm{R}=\frac{\mathrm{F}(60 \mu \mathrm{m})}{\mathrm{F}(100 \mu \mathrm{m})}$
$\mathrm{F}$ (60 micron) and $\mathrm{F}(100$ micron) are the flux densities at and 10 , respectively. In this way we can use equation (1) for the determination of the dust grain temperature ( Dupac et al. 2003).

\subsection{Dust Mass Estimation}

The dust masses are estimated from the IR flux densities. The resulting dust mass depends on the physical and chemical properties of the dust grains, the adopted dust temperature $T_{d}$ and the distance D to the object . (Hildebrand 1983)

$$
M_{\text {dust }}=\frac{4}{3} \frac{a \rho}{Q_{v}}\left[\frac{S_{v} D^{2}}{B(v, T)}\right]
$$

Where, $\alpha=$ Weighted grain size $=0.1 \mu \mathrm{m}$

$\rho=$ Grain density $=3000$

$Q_{v}=$ grain emissivity $=0.0010$ for $100 \mu \mathrm{m}$ and 0.0046 for $60 \mu \mathrm{m}$ respectively (Young et al. 1993)

$S_{v}=$ total flux density of the region whose mass is to be determined

\section{$S_{v}=f \times 5.288 \times 10^{-9} M J y / S r$}

$D=$ distance of the structure

$B(v, T)=$ Planck's function (Beichman et al. 1988).

$B(v, T)=\frac{2 h v^{3}}{c^{2}}\left[\frac{1}{e^{\frac{h v}{k T}}-1}\right]$

$h=$ Planck's constant

$c=$ velocity of light

$v=$ frequency at which the emission is observed

$T=$ the average temperatures of the region.

Values of different parameters we use in the calculation of the dust mass in our interest are as follows:

Using these values the equation (3) takes the form (Hildebrand 1983):

$M_{\text {dust }}=0.4\left[\frac{S_{v} D^{2}}{B(v, T)}\right]$

We use the equation for the calculation of the dust mass. It is clear from the expression (4) that the value of Planck function $B(v, T)$ for longer wavelength is higher than that of the shorter wavelength. Consequently, the range of $B(v, T)$ for fixed temperature (say $\Delta \mathrm{T}$ ) goes narrower if wavelength of the images increases. (Beichman et al. 1988). 


\subsection{Outflow}

To estimate the outflow nature of C - rich AGB star we use following relation. It is straight forward to show that, for a stationary polytropic wind,

$u_{\text {out }}^{2}=\frac{2}{\gamma-1} C_{s}^{2}\left(R_{c}\right)+(\Gamma-1) u_{e s c}^{2}\left(R_{c}\right)$

where $\mathrm{u}_{\mathrm{esc}}$ is the escape speed, $\mathrm{c}_{\mathrm{s}}$ is the sound speed, $\mathrm{R}_{\mathrm{c}}$ is the condensation radius and is the polytropic index in a polytropic equation of state. For the mass - loss rate, the correlation with $\Gamma$ (or) is weaker, which is due to variations of the gas-density profiles of the wind region between different models. ( Mattsson et al. 2010)

$$
\tilde{A}=\frac{\hat{e} L_{\star}}{4 \pi c G M_{\star}}
$$

Where

$\kappa=$ Total flux Mean Opacity

$L_{\star}=$ Stellar luminosity

$M_{\star}=$ Stellar mass

$\mathrm{c}=$ speed of light and $\mathrm{G}=$ constant of gravity.

On the process of calculation of $\mathrm{u}_{\text {out }}$ we assume polytropic index for $\mathrm{AGB}$ region $=1.5$,

and $\kappa=1 \mathrm{~cm}^{2} \mathrm{~g}^{-1}=0.1 \mathrm{~m}^{2} \mathrm{Kg}^{-1}$ for $100 \mu \mathrm{m}$ emitter source (Ossenkopf et al. 1994)

\subsection{Energy Estimation}

To estimate the energy of the region of the interest we assume here that the our targeted energy values are those with this energy value mass of central region moving outward. The outer wind expands quietly at a constant velocity. This is by far the largest part of the wind (extending up to 1000 - $100000 \mathrm{R}_{\star}$ depending on the mass-loss history) with temperatures decreasing to $\sim 10 \mathrm{~K}$. We use calculated value of average mass difference per pixel. With the help of classical kinetic energy relation we are focused to estimate the values of outflow energy.

$E=\frac{1}{2} M_{\text {deficit }} u_{\text {out }}^{2}$

\section{RESULT AND DISCUSSION}

Fig. 2 shows dust structures of both candidates at 100 microns. The contour levels are shown. The lines AB \& CD represent the major and minor diameters passing through minimum of flux density (flux-minima). The line EF is the line joining minimum flux and minimum temperature.
Optimum isocontours for candidates I and II are found at levels 41 and 52 respectively.

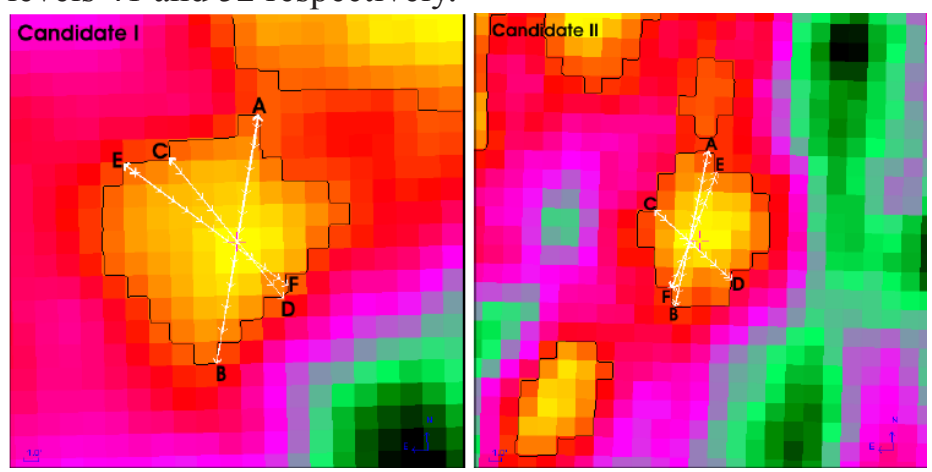

Figure 2: Left for R.A./Dec. J2000): $04^{h} 46^{m} 09^{s} /+32^{\circ} 28^{\prime} 00^{\prime \prime}$ and Right for R.A./Dec.(J2000): $05^{h} 06^{m} 02^{s} /+40^{\circ} 40^{\prime} 00^{\prime \prime}$, here Line AB Representing major diameter CD Minor diameter and EF distance which passes through minimum temperature pixel and minimum flux pixel respectively.

\subsection{Flux Density Variation}

By using the Aladin v 2.5 and Aladin v 8.0 software, flux density variation of the region of interest is studied. We obtained the graph of flux density variation along the major diameter, minor diameter and line joining the minimum temperature region and minimum relative flux region considering cavity as centre. As we move from point A we can see that the decrease in flux slowly at first, after certain distance the flux decrease rapidly to reach the minima point. After reaching minima the flux increase rapidly at first and then slowly with other two secondary minima with little bump. This feature indicate that the mass is expelling by some means from the central of cavity region. The best fit polynomial (5th degree polynomial for both Candidate I and II) are given by the equations

$$
\begin{aligned}
& S_{v}=13.6-37.9 d+93.6 d^{2}-194.3 d^{3}+195.2 d^{4}-65.2 d^{5} \\
& S_{v}=8.9-2.5 d+6.2 d^{2}-9.1 d^{3}+6.9 d^{4}-1.9 d^{5}
\end{aligned}
$$
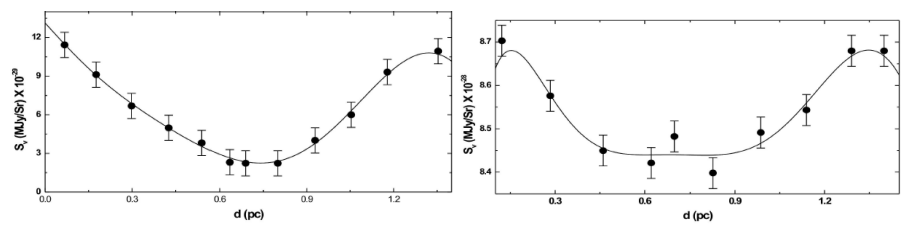

Figure 3: Curve left for cavity: R.A./Dec.(J2000): 04h 46m 09s I +32028' 00" and curve right for cavity R.A./Dec.(J2000): 05h

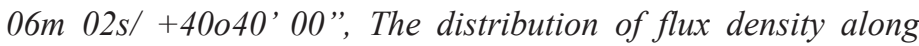
major diameter $A B$. The solid circle with error bars represent the standard error of the distribution. The solid curves represent the best fitt polynomial (5th order polynomial) respectively. 

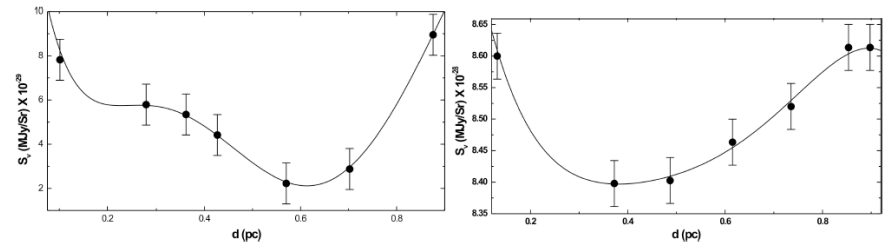

Figure 4: The distribution of temperature along minor diameter CD. Curve left for cavity: R.A./Dec.(J2000): $04^{h} 46^{m} 09^{s} /+32^{\circ} 28^{\prime}$ 00" and curve right for cavity R.A./Dec.(J2000): $05^{\mathrm{h}} 06^{\mathrm{m}} 02^{\mathrm{s}} /$ $+40^{\circ} 40^{\prime} 00^{\prime}$. The solid circle with error bars represent the standard error of the distribution. The solid curves represent the best fit polynomial (5th order polynomial) respectively.

$\mathrm{S}_{\mathrm{v}}=17.9-173.4 \mathrm{~d}+936.4 \mathrm{~d}^{2}-2316.4 \mathrm{~d}^{3}+2533.1 \mathrm{~d}^{4}-$ $989.0 \mathrm{~d}^{5}$

For candidate II the variation of relative density with distance give a best fit polynomial of order 5 is given below.

$S_{v}=9.2-7.5 d+27.2 d^{2}-50: 4 d 3+47.6 d^{4}-17.6 d^{5}$
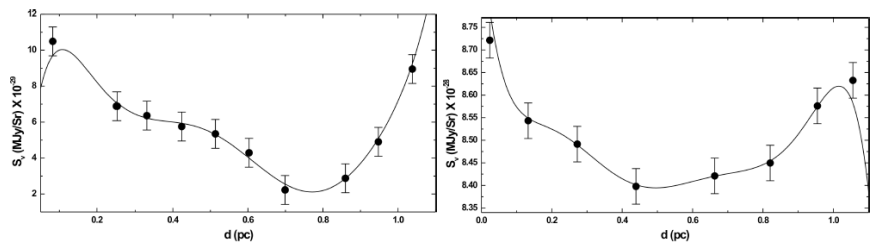

Figure 5: Curve left for cavity: R.A./Dec.(J2000): $04^{h} 46^{m} 09^{s} /$ $+32^{\circ} 28^{\prime} 00^{\prime \prime}$ and curve right for cavity R.A./Dec.(J2000): $05^{h} 06^{m}$ $02^{s} /+40^{\circ} 40^{\prime} 00^{\prime \prime}$, The distribution of flux density along the line joining the minimum temperature and minimum flux $(E F)$ is shown in graph. The solid circle with error bars represent the standard error of the distribution. The solid curves represents the best fit polynomial of 5 th order for both candidates (I and II). A very good agreement between Gaussian curve and best fitted line can be seen so we are neglect gaussian curve in both the cases.

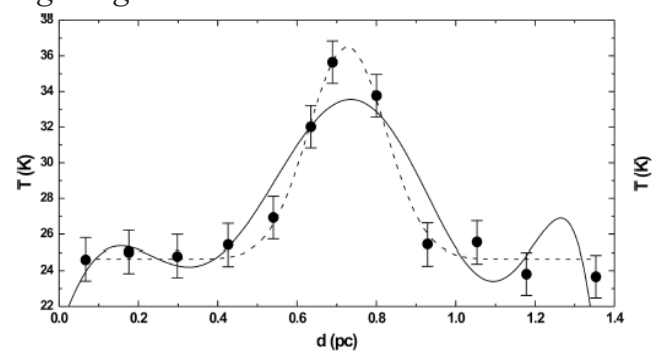

Figure 6: The distribution of temperature along major diameter $A B$ of both region. Graph left representing R.A./Dec.(J2000): 04 $46^{m} 09^{s} /+32^{\circ} 28^{\prime} 00^{\prime \prime}$ and curve right for cavity R.A./Dec.(J2000):

$05^{h} 06^{m} 02^{s} /+40^{\circ} 40^{\prime} 00^{\prime \prime}$, The solid circle with $\pm \frac{\sigma}{\sqrt{n}}$ error bars represent the standard error of the distribution. The solid, and the best fit polynomials are $5^{\text {th }}$ and $7^{\text {th }}$ order polynomial respectively. $\mathrm{S}_{\mathrm{v}}=12.6-17.1 \mathrm{~d}-182.0 \mathrm{~d}^{2}+1092.3 \mathrm{~d}^{3}-2303.2 \mathrm{~d}^{4}+2067.3 \mathrm{~d}^{5}$ $-662.5 \mathrm{~d}^{6}$
For candidate II there is best fitted polynomial is 7 th order polynomial which is given below:

$\mathrm{S}_{v}=8.9-7.1 \mathrm{~d}+63.9 \mathrm{~d}^{2}-292.0 \mathrm{~d}^{3}+698.4 \mathrm{~d}^{4}-896.9 \mathrm{~d}^{5}+$ $585.9 \mathrm{~d}^{6}-153.0 \mathrm{~d}^{7}$

From above it is clear that the relative flux density variation is clearly observed first of all decreases slowly after that rapid variation of flux observed up to central region. And with some fluctuations there are symmetrical behavior observed.

\subsection{Dust Color Temperature}

Using the method of Schnee et al. (2005) we calculated dust color temperature of each pixel inside both minima lying in the region of interest. We use the IRAS $100 \mu \mathrm{m}$ and 60 $\mu \mathrm{m}$ FITS images downloaded from the IRAS server. For the calculation of temperature we choose the value of $=2$ following the explanation given by Dupac et al. (2003). The region with minimum and maximum temperature is found to lie in the range of $21.65 \mathrm{~K}$ to $35.64 \mathrm{~K}$ and for second interested region $18.51 \mathrm{~K} 19.29 \mathrm{~K}$. It is very interesting for candidate I offset temperature of dust $=13.99 \mathrm{~K}$ and for other region offset temperature $=0.78 \mathrm{~K}$. There may be physics behind this may be due to in candidate I there is asymmetrical behavior is found. But in case of lower temperature variation case there is symmetric outflow or symmetric distribution of density and temperature. For candidate I

$\mathrm{T}=21.0+70.5 \mathrm{~d}-256.7 \mathrm{~d}^{2}-482.8 \mathrm{~d}^{3}+3842.1 \mathrm{~d}^{4}-6667.8 \mathrm{~d}^{5}$ $+4668.3 d^{6}-1169.6 d^{7}$

For candidate II the best fitted polynomial is 7 th order polynomial shown as:

$\mathrm{T}=17.1+30.1 \mathrm{~d}-193.5 \mathrm{~d}^{2}+575.3 \mathrm{~d}^{3}-906.3 \mathrm{~d}^{4}+779.6 \mathrm{~d}^{5}-$ $345.5 \mathrm{~d}^{6}+61.7 \mathrm{~d}^{7}$
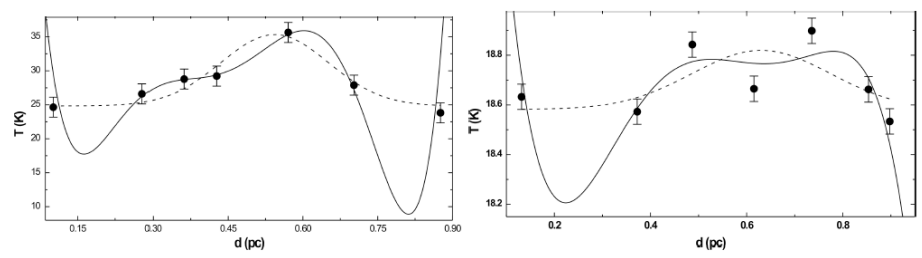

Figure 7: The distribution of Temperature along minor diameter CD. Graph left representing R.A./Dec.(J2000): $04^{h} 46^{m} 09^{s} /$ $+32^{\circ} 28^{\prime} 00^{\prime}$ " and curve right for cavity R.A./Dec.(J2000): $05^{h} 06^{m}$ $02^{5} /+40^{\circ} 40^{\prime} 00^{\prime \prime}$, The solid circle with error bars represent the standard error of the distribution. The solid represent the best fit polynomial are 5 th and 6 th order polynomials respectively.

$\mathrm{T}=-14.8+725-5 \mathrm{~d}-4355.7 \mathrm{~d}^{2}+11578.7 \mathrm{~d}^{3}-13694.0 \mathrm{~d}^{4}+58$ $66.3 d^{5}$ 
For candidate II along minor diameter the best fitted polynomial is of the form

$\mathrm{T}=54.7-570.8 \mathrm{~d}+3201.3 \mathrm{~d}^{2}-8676.3 \mathrm{~d}^{3}+12335.9 \mathrm{~d}^{4}-$ $8862.2 \mathrm{~d}^{5}+2538.3 \mathrm{~d}^{6}$

For candidate I the best fitted polynomial is 7 th order polynomial is of the form:

$\mathrm{T}=73.1-1240.0 \mathrm{~d}+10234.0 \mathrm{~d}^{2}-39728.9 \mathrm{~d}^{3}+82197.7 \mathrm{~d}^{4}$ $-926323.0 d^{5}+53497.3 d^{6}-12377.3 d^{7}$

For candidate II best fit polynomial is given by equation:

$\mathrm{T}=18.4+7.2 \mathrm{~d}-57.8 \mathrm{~d}^{2}+169.2 \mathrm{~d}^{3}-191.9 \mathrm{~d}^{4}+21.4 \mathrm{~d}^{5}+$

$101.6 d^{6}-49.4 d^{7}$

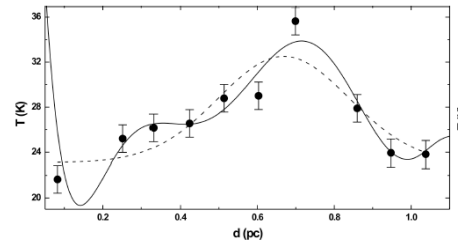

Figure 8: The distribution of line joining minimum flux pixel and minimum temperature pixel. Graph left representing R.A./ Dec.(J2000): 04h $46^{m} 09^{s} /+32^{\circ} 28^{\prime} 00^{\prime \prime}$ and curve right for cavity. RA./Dec.(J2000): $05^{h} 06^{m} 02^{s /}+40^{\circ} 40^{\prime} 00^{\prime \prime}$, The solid circle with error bars represent the standard error of the distribution. The solid curves represent and the best fit polynomial 7th order polynomials for both (I, II).

The region in which minimum and maximum temperature is found to lies in the range of $21.649 \mathrm{~K}$ to $35.644 \mathrm{~K}$ and for second interested region $18.507 \mathrm{~K}$ to $19.289 \mathrm{~K}$. It is very interesting for candidate I offset temperature of dust $=13.994 \mathrm{~K}$ and for other candidate offset temperature $=$ $0.783 \mathrm{~K}$. There may be physics behind this may be due to in candidate I there is asymmetrical behavior is found. But in case of lower temperature variation case there is symmetric outflow or symmetric distribution of density and temperature. An offset of about $14 \mathrm{~K}$ and $1 \mathrm{~K}$ suggest that our structure is not independently evolved or the role of discrete point sources in the field of cavity is important for the structure destruction mechanism. The cavity may be in thermally pulsating phase. The dust color temperature less than $20 \mathrm{~K}$ represents the interstellar cirrus cloud. Thus one of our far infrared dust structure (i.e. Cavity) is not a cirrus cloud. Another region of cloud fulfills the criteria of Cirrus clod. The average temperature of the dust is $24.833 \mathrm{~K}$ and $18.825 \mathrm{~K}$ respectively.
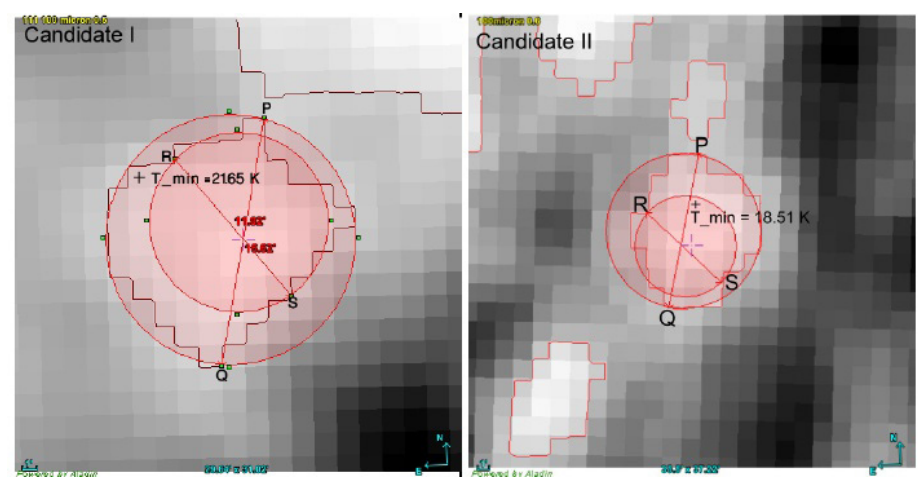

Figure 9: Left for R.A./Dec. (J2000): $04^{h} 46^{m} 09^{S} /+32^{\circ} 28^{\prime} 00^{\prime \prime}$ and Right for RA/DE(J2000): $05^{h} 06^{m} 02^{s} /+40^{\circ} 40^{\prime} 00^{\prime \prime}$, here Line $P Q$ representing major diameter $R S$ representing minor diameter respectively. Here the circle drown are assigned as inner and outer circle used for observation of outflow nature of $A G B$ wind.

Table 1: The table shows the Sum, Average, and mass per Pixel of region of Interest

\begin{tabular}{|c|c|c|c|}
\hline Region & $\begin{array}{c}\text { Total mass } \\
(\mathrm{Kg})\end{array}$ & $\begin{array}{c}\text { Average } \\
\text { Mass }(\mathrm{Kg})\end{array}$ & $\begin{array}{c}\text { Average Mass } \\
\text { Difference } \\
(\mathrm{Kg})\end{array}$ \\
\hline Inner Circle (I) & $3.182 \times 10^{25}$ & $6.770 \times 10^{23}$ & \\
Outer Circle (I) & $1.205 \times 10^{26}$ & $2.490 \times 10^{24}$ & $1.810 \times 10^{24}$ \\
Inner Circle (II) & $1.087 \times 10^{27}$ & $5.177 \times 10^{25}$ & \\
Outer Circle (II) & $2.587 \times 10^{27}$ & $1.000 \times 10^{26}$ & $4.656 \times 10^{25}$ \\
\hline
\end{tabular}

\section{AGB OUTFLOW}

Table 2: The table shows the sound speed (cs), escape speed (uesc), and wind speed (uout), i.e., outflow.

\begin{tabular}{|l|l|l|l|}
\hline \multicolumn{1}{|c|}{ Region } & \multicolumn{1}{c|}{$\mathrm{Cs}\left(\mathrm{ms}^{-1}\right)$} & $\mu_{\text {esc }}\left(\mathrm{ms}^{-1}\right)$ & $\mu_{\text {out }}\left(\mathrm{ms}^{-1}\right)$ \\
\hline Inner Circle (I) & 6.486 & 0.532 & 12.962 \\
Inner Circle (II) & 5.567 & 3.146 & 10.710 \\
\hline
\end{tabular}

Table 3: The table shows the, wind speed (uout) and their corresponding energy value $\mathrm{s}$

\begin{tabular}{|l|l|l|c|}
\hline \multicolumn{1}{|c|}{ Region } & \multicolumn{1}{c|}{$\mathrm{M}_{\text {deficit }}(\mathrm{Kg})$} & $\mathrm{u}_{\mathrm{esc}}\left(\mathrm{ms}^{-1}\right)$ & $\mathrm{E}(\mathrm{J})$ \\
\hline Candidate I & $1.810 \times 10^{24}$ & 12.962 & $1.173 \times 10^{25}$ \\
Candidate II & $4.8232 \times 10^{27}$ & 10.710 & $2.766 \times 10^{27}$ \\
\hline
\end{tabular}

\section{CONCLUSION}

The size of the structure are $1.354 \mathrm{pc} \times 0.971 \mathrm{pc}$ for candidate I and $1.424 \mathrm{pc} \times 0.925 \mathrm{pc}$ for candidate II where, major and minor diameter is found to be $1.354 \mathrm{pc}$ and 0.971 pc for candidate I and that of candidate II are $1.424 \mathrm{pc}$ and $0.925 \mathrm{pc}$ respectively. The minimum flux region is found to be located at center of lower part, at R.A. (J2000) = 
04o46m13.84s, Dec.(J2000) = +32o31' 39.6" for candidate I and $05 \mathrm{~h} 05 \mathrm{~m} 59.58 \mathrm{~s},+40 \mathrm{o} 40$ '33.4" for Candidate II respectively. A study of distance versus flux density, temperature variation shows a prominent condensation of dust at $100 \mu \mathrm{m}$ image and Gaussian-like distribution is observed along major and minor diameters of the structure. In addition, similar Gaussian-like distribution is found along line joining the minima and the lower temperature region. Gaussian-like distribution

suggests that the cavity structure is less disturbed from the external factors, i.e., possibility symmetric phenomena. A study of outflow suggests that the outflow velocity of structure is not uniform and not regularly shaped. The dust color temperature is found to lie in the range $21.65 \mathrm{~K}$ to $35.64 \mathrm{~K}$ for candidate I and $18.51 \mathrm{~K}$ to $19.29 \mathrm{~K}$ for candidate II, with an offset of about $14 \mathrm{~K}$ for candidate I and $0.8 \mathrm{~K}$ for candidate II. An offset $14 \mathrm{~K}$ suggests that the AGB is hot in the early stage. We expect deviation from symmetrical outflow, i.e., AGB wind probably prefer polytropic behavior implies bipolar type wind. Whereas the offset $0.8 \mathrm{~K}$ for candidate II suggests that the AGB is in symmetric AGB phase, i.e., early AGB stage. The total mass of the dust in the cavity structure is found about $5.9310^{25} \mathrm{Kg}\left(0.00003 \mathrm{M}_{\odot}\right)$ for candidate I and $1.9510^{27} \mathrm{Kg}\left(0.001 \mathrm{M}_{\odot}\right)$ for candidate II. We obtained energy of dissipation as $1.1710^{25} \mathrm{~J}$ and 2.77 $10^{27} \mathrm{~J}$ for candidate I and II respectively which suggest that the energy of the order $10^{25}$ and $10^{27}$ Joules falls with in the limit of AGB wind energy. Hence from above observation it is concluded that there is asymmetrical or aspherical phenomena observed. Above result suggest that there is somewhat polytropic behavior occur. Also there is further study needed for solve such problem.

\section{ACKNOWLEDGEMENTS}

We acknowledge Prof. Dr. Binil Aryal ,CDP,TU ( our supervisor), for proper guideline \& supervision of this research work. We are also indebted to Prof. Ronald Weinberger of Innsbruck University, Innsbruck, Austria, for providing substantial data. This research has made use of NASA/IPAC Extragalactic Database (NED) which is operated by the Jet Propulsion Laboratory, California
Institute of Technology, under contract with the National Aeronautics and Space Administration.

\section{REFERENCES}

[1] I. J. Iben \& A. Renzini, A\&A 21, 271 (1983)

[2] S. Hoefner \& E. A. Dorfi, A\&A 319, 648 (1997)

[3] T. Bloecker \& D. Schoenberner, A\&A 244, L43 (1991)

[4] B. Aryal, C. Rajbahak, R. Weinberger, MNRAS 402, 1307 (2010)

[5] T. Padmanabhan, Theoretical Astrophysics, Vol. II, p. 469, $1^{\text {st }}$ South Asian edition, Cambridge university Press, New Delhi (2010)

[6] K. W. Suh \& Y. J. Kwon, JKAS 42, 81 (2009).

[7] http://skyview.gsfc.nasa.gov/current/cgi/query.pl (SkyView, 2014))

[8] http://simbad.u-strasbg.fr/simbad/ (SIMBAD, 2014)

[9] S.F., Odenwald, Astrophysical Journal. 325, 320 (1988)

[10] S. L. Schnee, N. A. Ridge, A. A. Goodman, G. L. Jason, ApJ 634, 442 (2005)

[11] X. Dupac, J. P. Bernard, N. Boudet, M. Giard, J. M. Lamarre, C. Mny, F. Pajot, I. Ristorcelli, G. Serra, B.

Stepnik, J. P. Torre, A\&A 404, L11 (2003)

[12] R. H. Hildebrand, QJRAS 24, 267 (1983)

[13] K. Young, T. G. Phillips, G. R. Knapp, ApJ 409, 725 (1993)

[14] R. Weinberger, Private Communication, (2014)

[15] C. A. Beichman, Neugebauer G., H. J. Habing, P. E. Clegg, T. J. Chester, Infrared Astronomical Satellite (IRAS) Catalogues and Atlases I: Explanatory Supplement. US Government Printing Office, Washington, (1988)

[16] L. Mattsson, R. Wahlin and S. Hoefner, A\&A 509, A14, 13 (2010)

[17] V. Ossenkopf \& Th. Henning, A\&A 291, 943 (1994) 68 